\title{
Migraine and risk of cardiovascular disease in women: prospective cohort study
}

In this Research paper by Kurth and colleagues (BMJ 2016;353:i2610, doi:10.1136/bmj.i2610), the authors wish to clarify that the information on migraine and all other covariates during follow-up was updated in the main analyses. Thus, the analysis included not only the 17531 women who reported migraine at baseline, but also the status change of the 6389 women who reported migraine during follow-up.
Furthermore, Rimma Dushkes, JoAnn E Manson, and Kathryn M Rexrode are affiliated not with the Institute of Public Health, Charité - Universitätsmedizin, Berlin, Germany, but with the Division of Preventive Medicine, Department of Medicine, Brigham and Women's Hospital, Harvard Medical School, Boston, MA, USA. Walter C Willett is affiliated additionally with Channing Division of Network Medicine, Department of Medicine, Brigham and Women's Hospital, Harvard Medical School, Boston, MA, USA. 